\title{
Down-regulation of EDAG expression by retrovirus-mediated small interfering RNA inhibits the growth and IL-8 production of leukemia cells
}

\author{
BIN LING ${ }^{1,2}$, YING ZHOU ${ }^{1,2}$, DINGQING FENG ${ }^{1,2}$, GUODONG SHEN ${ }^{1,2}$, \\ TING GAO $^{1,2}$, YONGYUN SHI ${ }^{1,2}$, HAIMING WEI ${ }^{3}$ and ZHIGANG TIAN ${ }^{3}$ \\ ${ }^{1}$ Anhui Province Key Laboratory of Molecular Medicine; ${ }^{2}$ Anhui Provincial Hospital Affiliated to Anhui \\ Medical University, Hefei 230001; ${ }^{3}$ Hefei National Laboratory for Physical Sciences at Microscale and \\ School of Life Sciences, University of Science and Technology of China, Hefei 230027, P.R. China
}

Received January 26, 2007; Accepted May 25, 2007

\begin{abstract}
Embryonic development associated gene (EDAG), which is overexpressed in hematopoietic neoplasms and leukemia cell lines, has been reported to participate in the leukemia cell differentiation and proliferation. This study investigated whether retrovirus-mediated transfer of a siRNA against EDAG can reduce the growth of leukemia cells which highly express EDAG in vitro and in vivo. The stable transfected cells were identified with RT-PCR, the effect of EDAG/siRNA on the growth of the human erythroleukemia cell line HEL was analyzed by MTT assay, and angiogenic factor IL-8 release was evaluated by ELISA and RT-PCR. The results showed that EDAG/siRNA can silence the expression of EDAG in HEL cells. Down-regulation of EDAG expression by retrovirus-mediated siRNA inhibited the cell proliferation and tumor growth. Knockdown of EDAG expression by siRNA is also associated with decreased expression of the anti-angiogenic factor IL-8, suggesting that EDAG stimulates tumor growth at least in part by regulating angiogenesis. This study suggests that siRNA-mediated gene silencing of EDAG could potentially be a therapeutic strategy for EDAG over-expressing leukemia cells.
\end{abstract}

\section{Introduction}

Embryonic development associated gene (EDAG), which maps to chromosome $9 \mathrm{q} 22$, a region containing the break-

Correspondence to: Dr Bin Ling, Anhui Provincial Hospital Affiliated to Anhui Medical University, 17 Lu-Jiang Road, Hefei City, 230001 Anhui, P.R. China

E-mail: lingbin.ling@gmail.com

Abbreviations: IL-8, interleukin-8; NF-кB, nuclear factor-kappa B; AML, acute myeloid leukemia; ALL, acute lymphoblastic leukemia; FAB, French-American-British; siRNA, small interfering RNA

Key words: IL-8, EDAG, siRNA, leukemia points of several hematopoietic neoplasms, is mainly expressed in adult bone marrow $\mathrm{CD}_{34}{ }^{+}$progenitor cells, testis, and fetal liver. EDAG is also expressed highly in leukemia cells (HEL, K562, Dami, Meg-01 and MO7e), acute myeloid leukemia (AML) and acute lymphoid leukemia (ALL), which is considered to be involved in the process of leukemia cell differentiation and proliferation (1-4). Substantial evidence shows that EDAG is homologous to mouse Hemgn and rat RP59 (5), and plays roles in the hematopoietic differentiation. However, the mechanisms of how EDAG exerts biological functions remain unknown. Yang et al found that the expression of EDAG transcripts was closely correlated with levels of GATA1 transcripts in primary acute myeloid leukemia specimens, and GATA1 can regulate the molecular pathways of hematopoiesis through direct binding to the GATA site-dependent Hemgn promoter in mouse (6). Li et al also established that EDAG can regulate the proliferation and differentiation of hematopoietic cells and resist cell apoptosis through the activation of nuclear factor-kappa B (NF-кB) (7).

To date, it has been difficult to define the precise effect of EDAG expression in leukemia cells. A zinc-inducible antisense construct against EDAG showned that the proliferation and colony formation were decreased remarkably in cell line K-562 that highly expresses EDAG (7).The use of a zinc-inducible antisense construct needs the stimulator of $\mathrm{ZnSO}_{4}$, which restricts the study of tumor growth in vivo. In this study, we used retrovirus-mediated expression of siRNA against EDAG expression in models of human leukemia cell line with the goals of clarifying the inhibition of siRNA against EDAG and tumor growth in vitro and in vivo.

Interleukin-8 (IL-8), purified in 1987 by several groups using LPS stimulated peripheral blood mononuclear cells, is a CXC chemokine involved in neutrophil chemoattraction, angiogenesis, and stem cell mobilization. It is mainly expressed in activated monocytes and neutrophils. Moreover, AML, ALL and several solid tumor types also secrete IL-8, especially after stimulation with 1ipopolysaccharide (LPS) or 12-O-tetradecanoylphorbol-13-acetate (TPA) (8-10). Current 
Table I. Sequences of siRNA oligonucleotides.

\begin{tabular}{ll}
\hline RNA oligonucleotide & \multicolumn{1}{c}{ Sequence } \\
\hline siRNA & 5'-ggatccGCTCCTAACACATGCCAAGTttcaagagaACTTGGCATGTGTT \\
& AGGAGTTTTTTacgcgT gaattc----3'
\end{tabular}

siRNA-negative

5'-ggatccGTGCGTTGCTAGTACCAACttcaagagaTTTTTTacgcgTgaattc-3'

Lowercase letters indicate the loop nucleotides, italic lowercase letters are BamHI, MIUI, and EcoRI nucleotides, and bold italic uppercase letters indicate the sequence of the transcription terminator.

reports show that high level of IL-8 was predictive of poor survival in AML and ALL (11-13), and IL-8 production has been shown to be mediated by $\mathrm{NF}-\kappa \mathrm{B}$ in leukemia cells (14-16).

In this study, we constructed recombinant retrovirus plasmids to knockdown the expression of EDAG in HEL cells and identified stable transfected HEL cell lines, we found that cell proliferation, tumor growth and IL-8 release were inhibited after silencing the expression of EDAG in HEL cells.

\section{Materials and methods}

Materials and reagents. Retrovirus vector (RNAi-Ready pSIREN-RetroQ vector) was purchased from BD Biosciences and used for constructing the siRNA in the present study. MIuI, BamHI, EcoRI, T4 DNA ligase for construction and identification of the recombinant plasmid were obtained from Takara Bio Inc. Lipofectamine 2000 ${ }^{\mathrm{TM}}$ (Invitrogen), chloroquine (Sigma), and Polybrene (Sigma) were used for transfection. The reverse transcription kit was from Promega and the PCR kit was from Takara Bio Inc. DNA purification system from Promega was to extract the recombinant plasmids. Puromycin from BD Clontech was used to select stable transfection cell lines. Stimulation agent TPA was obtained from Sigma. MTT (Amresco) was provided to assay proliferation. IL-8 ELISA kit was purchased from R\&D Systems. Culture media and fetal bovine serum were from Gibcol Co. The primers were synthesized and E. coli $\mathrm{DH} 5 \alpha$ purchased from Shanghai Sangon Co., Ltd.

Viral vector construction. The siRNA sequence targeting EDAG gene was designed according to manufacturer's instruction and listed in Table I, which matches to the coding sequence of EDAG mRNA (with Genebank Accession number: NM:018437) located at 741 bp-759 bp downstream of start codon ATG. The synthesized EDAG siRNA oligos were annealed and inserted into pSIREN-RetroQ downstream of U6 promoter, and assigned as EDAG/siRNA. The constructs were identified by digestion of $M l u \mathrm{I}$, and further verified by sequence analysis.

Cell lines. The human erythroleukemia cell line HEL (ATCC) and retroviral packaging PT67 cells (BD) were cultured in complete RPMI-1640 containing 10\% fetal bovine serum respectively. All the cells were grown in medium supplemented with $100 \mathrm{U} / \mathrm{ml}$ penicillin and $100 \mathrm{U} / \mathrm{ml}$ streptomycin at $37^{\circ} \mathrm{C}$ in a moist atmosphere of $5 \% \mathrm{CO}_{2}$.
Retroviral infection. Recombinant retrovirus was packaged and produced according to manufacturer's instruction.

$1 \times 10^{5}$ PT67 cells were split into $35-\mathrm{mm}$ dishes to reach $60-80 \%$ confluency after $24 \mathrm{~h}$. Chloroquine $(25 \mu \mathrm{M})$ was added to medium $1 \mathrm{~h}$ prior to transfection. EDAG/siRNA vector were transfected into PT67 cells with Lipofectamine $2000^{\mathrm{TM}}$. Four hours after transfection, the plates were replaced the medium for the transfected cells with fresh complete medium, and $24 \mathrm{~h}$ later the cells were maintained in selective medium of $1.875 \mu \mathrm{g} / \mathrm{ml}$ puromycin to continue cultivation for 2-3 weeks. When the stable-transfected cells were established, the supernatant containing recombinant retrovirus particles was collected and filtered with a $0.45-\mu \mathrm{m}$ filter, and were used fresh. A negative control vector was also created in the parallel with EDAG/siRNA and assigned as EDAG/negative that contains an unrelated sequence to EDAG mRNA as listed in Table I. The same protocol was used to packaging and production of EDAG/negative retrovirus particles. HEL/siRNA and HEL/negative cell lines stably expressing siRNA were generated by transduction of HEL cells with retroviral containing EDAG/siRNA or EDAG/negative sequences, respectively. In briefly, HEL was cultured in $35-\mathrm{mm}$ plates to $70 \%$ of confluence. After removing the culture medium, the cells were washed twice with incomplete medium and incubated with $2 \mathrm{ml}$ of 1:1 mixture of virus supernatants and fresh incomplete culture medium containing $1.4 \mu \mathrm{g} / \mathrm{ml}$ polybrene for $1 \mathrm{~h}$. After infection twice, the infected cells were maintained with complete medium $24 \mathrm{~h}$, then the cells were selected with $1.875 \mu \mathrm{g} / \mathrm{ml}$ puromycin in the maintaining medium for 1 month and surviving cells (HEL/siRNA, HEL/ negative) were harvested for the study. The efficiencies of inhibiting activity by EDAG/siRNA were monitored by RTPCR for EDAG mRNA.

Analysis of EDAG expression level in siRNA-transfected cells by reverse transcription-PCR. Briefly, total RNA from each cell line was extracted with TRIzol according to the manufacturer's protocol (Invitrogen). RNA ( $1 \mu \mathrm{g})$ was reverse transcribed by random primer and M-MLV reverse transcriptase for $1 \mathrm{~h}$ at $37^{\circ} \mathrm{C} .5 \mu \mathrm{l}$ of 5 folds diluted cDNAs was then used as the templates for 30 cycles of PCR amplification with gene specific primers, and each cycle was performed at $94^{\circ} \mathrm{C}$ for $30 \mathrm{sec}, 56^{\circ} \mathrm{C}$ for $30 \mathrm{sec}$, and $72^{\circ} \mathrm{C}$ for $60 \mathrm{sec}$. PCR products were analyzed on $1 \%$ agarose gels. The primers used for PCR amplification were: EDAG sense, 5'-AAGCAC CATCAGACACCTGACC-3'; EDAG antisense, 5'-TGCTTG AAGAGAGCATCCTGCC-3'; $\beta$-actin sense, 5'-GTGGGGC GCCCCAGGCACCA-3'; ß-actin antisense, 5'-CTCCTTAAT GTCACGCACGATTT-3'. 


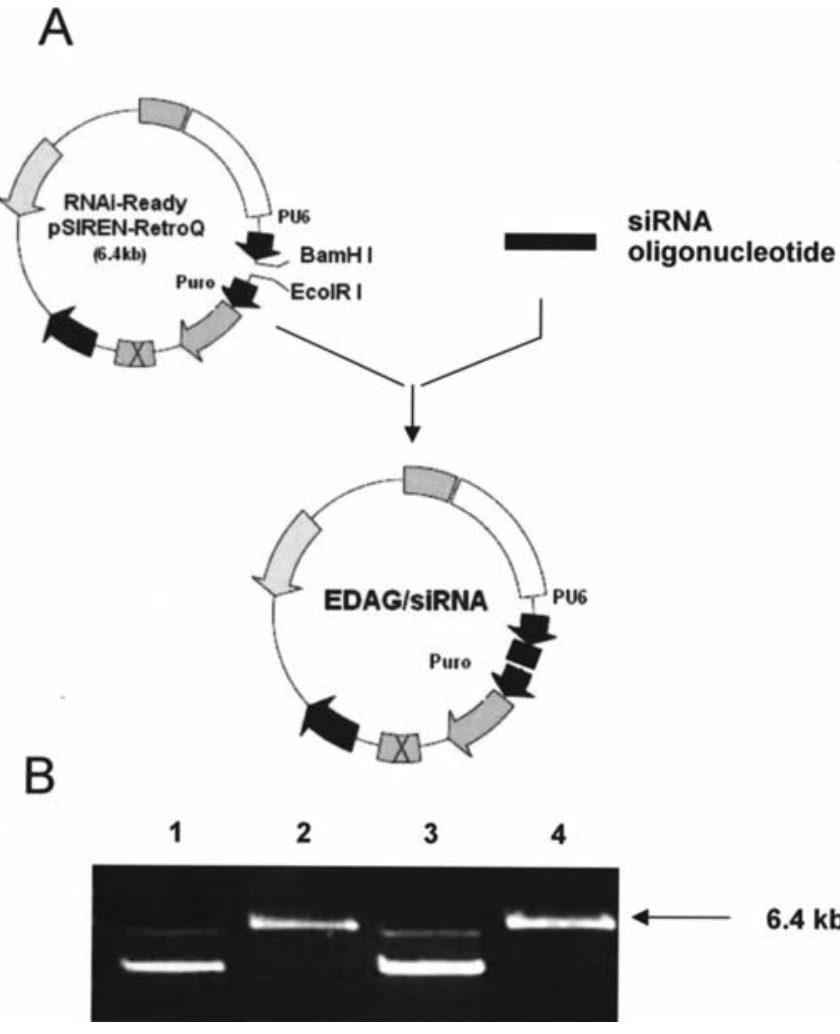

Figure 1. Construction and identification of recombinant retroviral plasmids. (A) The siRNA oligonucleotides were ligased into RNAi-Ready pSIRENRetroQ vector. (B) Identification of recombinant retroviral plasmids by restriction endonuclease $M I u \mathrm{I}$ digestion: $1 \mathrm{EDAG} /$ negative; $2 \mathrm{EDAG} /$ negative by $M I u \mathrm{I} ; 3 \mathrm{EDAG} / \mathrm{siRNA} ; 4 \mathrm{EDAG} / \mathrm{siRNA}$ by $M I u \mathrm{I}$ (the fragments of recombinant plasmids after digestion were the expected $6.4 \mathrm{~kb}$ ).

MTT assay. $1 \times 10^{4}$ cells were plated into 96-cell plate to incubate for 2,4 , and 6 days at $37^{\circ} \mathrm{C}$ in $5 \% \mathrm{CO}_{2}$ respectively. By the end of incubation, the plates were added $20 \mu 1 \mathrm{of} 5 \mathrm{mg} /$ ml MTT after discarding $100 \mu 1$ supernatant, and continued incubation at $37^{\circ} \mathrm{C}$ for $4 \mathrm{~h}$. After removing the dye solution, $100 \mu 1 \mathrm{DMSO}$ was added to each well and kept at room temperature for $30 \mathrm{~min}$, and then the absorbance rate at $570 \mathrm{~nm}$ was measured. All assays were performed in triplicate.

Stimulation of cells and quantitative determination of $I L-8$. Experiments were carried out in 24-well culture plates with starting cell concentrations of $5 \times 10^{5}$ cells $/ \mathrm{ml}$. Twenty-four hours after stimulation with or without $50 \mu \mathrm{g} / \mathrm{ml}$ TPA, the plates were centrifuged to collect the culture supernatants and cell pellets separately. The supernatants were stored frozen to inspect IL- 8 protein by ELISA, and the pellets were added with $500 \mu 1$ TRIzol to examine the expression of IL-8 mRNA. The primer sequences were: IL-8, 5'-ACTTCTCCACAACC CTCTGC-3' (sense); 5'-CCGGAAGGAACCATCTCACT-3' (antisense). The PCR program was as follows: $94^{\circ} \mathrm{C}$ for $30 \mathrm{sec}$, $56^{\circ} \mathrm{C}$ for $30 \mathrm{sec}$, and $72^{\circ} \mathrm{C}$ for $60 \mathrm{sec}$.

Animal experiments. The stable expressing cells HEL/negative $\left(2 \times 10^{6}\right)$ and HEL/siRNA were grown to confluence before harvested and resuspended in $0.1 \mathrm{ml}$ saline for subcutaneous administration. A total of 8 mice with $18-20 \mathrm{~g}$ body weight and 6 to 8 -week-old BALB/c athymic nude mice (Beijing

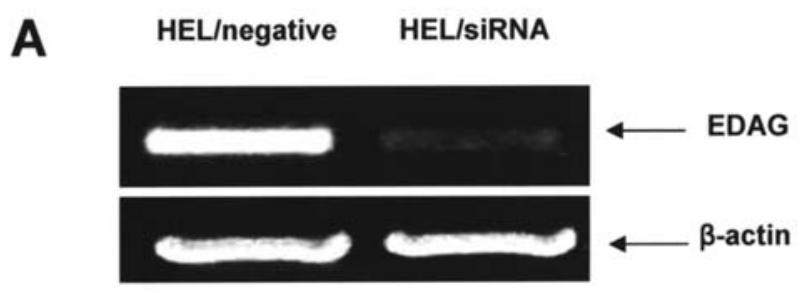

B

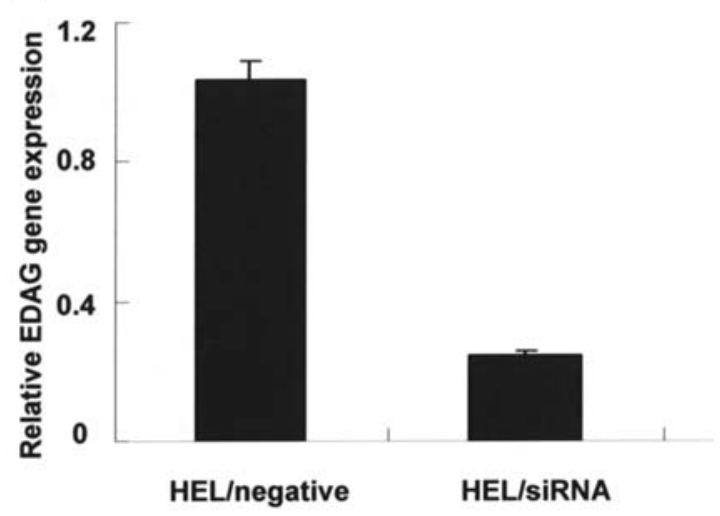

Figure 2. Semiquantitative PCR of EDAG mRNAs in HEL cells infected with EDAG/negative, EDAG/siRNA. (A) mRNA expression in HEL cells. HEL cells $\left(1 \times 10^{6}\right)$ were subjected to total cell RNA extraction and RT-PCR amplification. Reaction products were run on $1 \%$ agarose gel. In order to exclude the contamination, we also had a negative control group without the RT procedure. (B) Quantitative analysis of mRNA expression from A. $y$-axis indicates the rates of EDAG mRNA and actin mRNA.

experimental animal center) were divided into 2 groups for treatment and control groups with HEL/siRNA and HEL/ negative cells, respectively. The mice were observed every 10 days for 40 days after treatment and the tumor size were recorded. All the mice were housed in pathogen-free environments. All animal experiments were approved by the appropriate committee of University of Science and Technology of China. Tumor size was calculated using the formula described by Kyriazis et al (17): tumor volume $=$ width $^{2} \mathrm{x}$ length $\mathrm{x} 0.4$.

Statistical analysis. Results from at least three experiments were analyzed by SPSS13.0. The significance of the differences between two groups was assayed by independent t-test. For all tests, $\mathrm{p}$-values $<0.05$ were considered significant.

\section{Results}

Construction and identification of siRNA. RNA oligonucleotides were ligated into RNAi-Ready pSIREN-RetroQ vector (Fig. 1A), the recombinant plasmids were transformed into $E$. coli $\mathrm{DH} 5 \alpha$ to obtain renewable source of DNA. The desired plasmids (EDAG/siRNA, EDAG/negative) were identified by restriction analysis with $M I u \mathrm{I}$ for the expected fragments $6.4 \mathrm{~kb}$ (Fig. 1B), and we also confirmed the exact siRNA oligonucleotides by sequencing.

Expression of EDAG on HEL cells infected with EDAG/siRNA or EDAG/negative. We transfected PT67 cells with the EDAG/ 


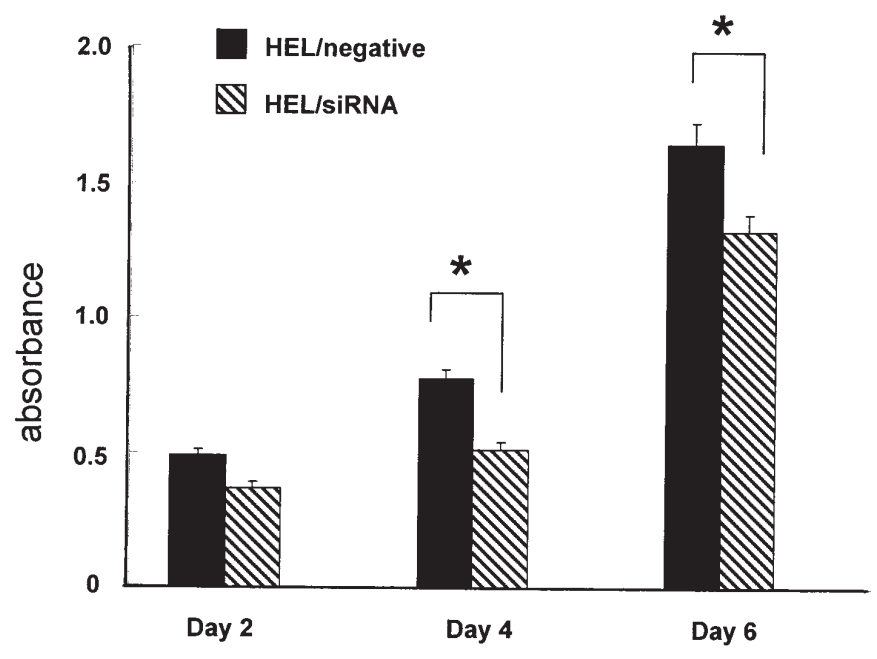

Figure 3. The proliferation of HEL is inhibited after infection with siRNAs. HEL cells $\left(1 \times 10^{4}\right)$ infected with EDAG/ negative, EDAG/siRNA were placed into a 96 -well plate and measured by MTT with a 570-nm filter after culture for 2,4 or 6 days $\left({ }^{*} \mathrm{P}<0.05\right.$ vs. HEL/negative).

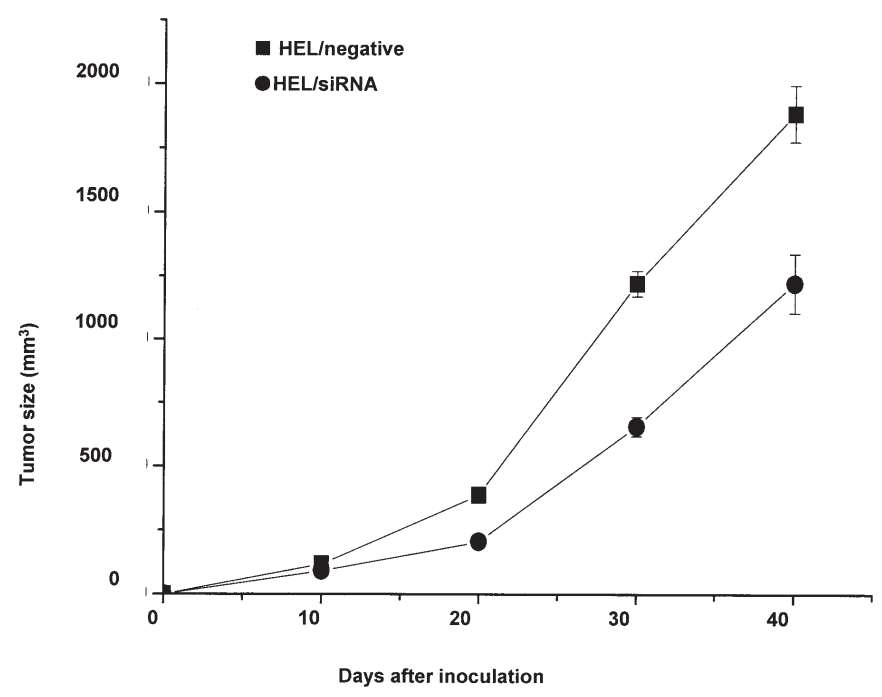

Figure 4. Tumor growth in HEL/negative or HEL/siRNA infected cells. HEL cells $\left(2 \times 10^{6}\right)$ infected with EDAG/negative, EDAG/siRNA were subcutaneously injected into nude mice bred in pathogen-free environments. The mice were checked every 10 days and sacrified at day 40 . Tumor growth at 40th day in a total of 4 mice analyzed was dramatically suppressed by EDAG/siRNA in HEL cells, compared with tumors from HEL/negative cells. ( ${ }^{*} \mathrm{P}<0.05$ vs. HEL/negative)

siRNA or EDAG/negative constructs to generate infectious retroviral particles that were subsequently used to infect the cell line HEL, in which EDAG is highly expressed. Cells were then selected in medium with puromycin and analyzed for the expression of EDAG. Cells infected with the recombinant retrovirus EDAG/siRNA showed markedly less EDAG mRNA (Fig. 2A and B), and the inhibition rate of EDAG/siRNA was $76.5 \%$.

The proliferation of HEL cells is inhibited by EDAG silencing. Since EDAG is known to stimulate cell growth (7), we examined the proliferation of HEL cells in which EDAG had
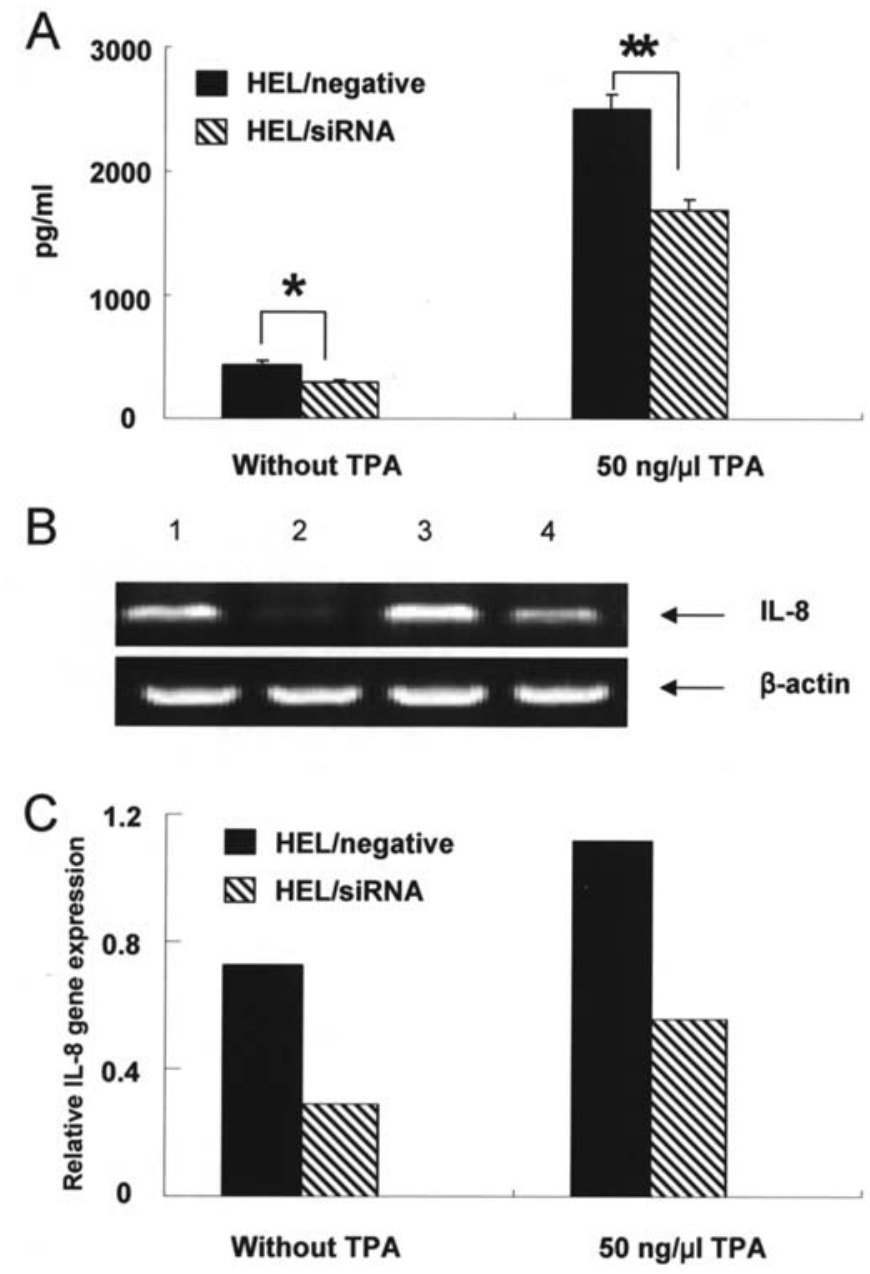

Figure 5. EDAG silencing inhibits the IL-8 release of HEL cells. HEL cells $\left(5 \times 10^{5}\right)$ were incubated for $24 \mathrm{~h}$ with or without TPA $(50 \mathrm{ng} / \mu \mathrm{l})$. (A) The IL-8 level in the supernatants was measured by ELISA, mean values of at least three independent experiments are indicated. ( ${ }^{*} \mathrm{P}<0.05$ vs. HEL/negative; * ${ }^{*}<0.01$ vs. HEL/negative). (B) IL- 8 mRNA levels by RTPCR assay. (C) Quantitative analysis of mRNA expression from B. y-axis indicates the rates of IL-8 mRNA and actin mRNA.

been silenced by retrovirus-mediated siRNA. Proliferation of $\mathrm{HEL} / \mathrm{siRNA}$ cells, as indicated by MTT, was substantially inhibited on day 4 and day 6 (p<0.05, Fig. 3$)$.

EDAG silencing inhibits the growth of tumor in vivo. In order to investigated whether anti-EDAG siRNA would affect tumor growth in vivo, HEL/negative and HEL/siRNA cells were injected subcutaneously into BALB/c nude mice. As shown in Fig. 4, HEL/siRNA showed strong suppression of tumor growth on day 40 compared with HEL/negative $(p<0.05)$. These results suggest that EDAG silencing inhibits the growth of tumors.

EDAG silencing inhibits the production of IL-8 in HEL cells. Several previous studies have demonstrated that IL-8 is also highly expression in AML and ALL, and can be regulated by $\mathrm{NF}-\kappa \mathrm{B}(10)$, we then investigated whether EDAG silencing can affect the IL- 8 production in HEL cells. As shown in Fig. 5, the expression of IL- 8 mRNA and protein was decreased in HEL cells infected with EDAG/siRNA. We then stimulated HEL cell lines with TPA (stimulator of IL-8) 
in vitro, the expression of IL-8 was notably enhanced, while IL-8 release was more significantly inhibited in HEL/negative cells $(\mathrm{p}<0.01)$. These results suggest that EDAG silencing can inhibit the production of IL-8 in HEL cells.

\section{Discussion}

EDAG, highly expressed in AML, ALL, has been showed to be involved in erythroleukemia differentiation, survival and cell transformation of NIH3T3 cells $(18,19)$. An et al has reported that initial expression of EDAG was closely associated with the response of malignant hematopoietic cells to chemotherapy (20). Li et al also observed that EDAG can resist cell apoptosis through the activation of NF-кB (6). All these studies suggest that overexpression of EDAG in acute leukemia plays an important role in leukemogenesis, and could be a new target in the treatment of leukemia. We have reported the expression of EDAG mRNA and protein in hematopoietic neoplasms and leukemia cell lines (HEL, K562) (4); in this study, we constructed retroviral recombinant plasmids with siRNA against EDAG, through retrovirus-mediated infection with recombinant plasmids. We found that EDAG silencing significantly reduced the expression of EDAG mRNA, inhibited cell proliferation and tumor growth, indicating that retrovirus mediated siRNA silencing is an effective approach for dissecting malignant signals in leukemia, and we need to explore the functions of EDAG through siRNA.

Angiogenesis seems to be important both for leukemogenesis and susceptibility to intensive chemotherapy, and antiangiogenic strategies are therefore considered for the treatment of AML and ALL. IL-8, known to act as a potent neutrophil chemoattractant, is able to participate in the control of hematopoietic cell proliferation and differentiation $(5,21,22)$, and it is important in human AML and ALL as a proangiogenic mediator $(11-13,23)$. IL-8 produced by the majority of AML with monocytic components [FrenchAmerican-British (FAB) M4 and M5] usually responds poorly to therapy in terms of long-term leukemia control (24-26). Herein we found that IL-8 production was decreased when the expression of EDAG in HEL was inhibited. Myeloid and lymphoid leukemia cells and leukemia cell lines (HEL, K562) spontaneously released IL-8, both constitutively and in response to TPA. These findings demonstrate that overexpression of EDAG strongly positively regulates IL-8 expression in hematopoietic cells, and reduction in EDAG levels using siRNA leads to a significant reduction of IL-8 levels in HEL that express both EDAG and IL-8, supporting a critical role for EDAG in IL-8 regulation. Expression of EDAG by leukemia cells increased IL-8 expression, stimulated angiogenesis, and enhanced leukemia cell growth and survival in the marrow microenvironment. Therefore, inhibition of the expression of EDAG might diminish the malignant biological behavior of leukemia cells by decreasing the production of IL-8.

The major finding of constitutive activation of $\mathrm{NF}-\kappa \mathrm{B}$ in leukemia stem cells and other neoplasms suggested that blocking $\mathrm{NF}-\kappa \mathrm{B}$ might be a potential strategy to treat leukemia/lymphoma (27-28). Li et al showed that NF-кB is a crucial mediator of the antiapoptotic signal delivered by
EDAG through transfecting EDAG into $\mathrm{Ba} / \mathrm{F} 3$ cells (7). The current study indicated that NF- $\mathrm{B}$ regulates IL- 8 release by $\mathrm{PTK} \rightarrow \mathrm{MAPK} \rightarrow \mathrm{NF}-\kappa \mathrm{B} \rightarrow \mathrm{IL}-8$ pathway $(10,15,29)$. According to the actions of EDAG on NF- $\kappa$ B, we propose that IL-8 production might be also mediated by the activation of NF- $\mathrm{\kappa B}$ which is positive relative with the expression levels of EDAG.

Collectively, the major finding of the current study is that the overexpression of EDAG in leukemia cells increases proliferation and contributes to the growth of neoplasms, which is likely to be mediated by activation of NF- $\mathrm{KB}$ and increasing the release of IL-8. Furthermore, the inactivation of EDAG may lead to the sensitization of leukemia cells to chemotherapeutic drug-induced differentiation and apoptosis. EDAG appears to play an important role in regulating IL-8 production, which acts on the malignant behavior of leukemia. As a modulator role for EDAG in leukemia, EDAG might be a new target in the treatment of leukemia.

\section{Acknowledgements}

This study was supported by the Chinese National Natural Science Foundation project (\#30471805), the Natural Science Foundation of Anhui Province, China (\#20050430715), the Natural Science Foundation of Anhui Provincial Educational Department, China (\#2006KJ080C).

\section{References}

1. Yang LV, Nicholson RH, Kaplan J, Galy A and Li L: Hemogen is a novel nuclear factor specifically expressed in mouse hematopoietic development and its human homologue EDAG maps to chromosome 9q22, a region containing breakpoints of hematological neoplasms. Mech Dev 104: 105-111, 2001.

2. Lu J, Xu WX, Wang SY, Zhan YQ, Jiang Y, Cai WM and Yang XM: Isolation and characterization of EDAG-1, a novel gene related to regulation in hematopoietic system. Sheng Wu Hua Xue Yu Sheng Wu Wu Li Xue Bao 33: 641-646, 2001.

3. Yang LV, Heng HH, Wan J, Southwood CM, Gow A and Li L: Alternative promoters and polyadenylation regulate tissuespecific expression of Hemogen isoforms during hematopoiesis and spermatogenesis. Dev Dyn 228: 606-616, 2003.

4. Zhou Y, Xu WX, Zhan YQ, Li CY, Xu CW, Zheng H, Li FF, Yang XM and Wang SY: Expression of EDAG-1 gene in human leukemia and lymphoma cell lines. Ai Zheng 23: 1238-1243, 2004 (In Chinese).

5. Kruger A, Ellerstrom C, Lundmark C, Christersson C and Wurtz T: RP59, a marker for osteoblast recruitment, is also detected in primitive mesenchymal cells, erythroid cells, and megakaryocytes. Dev Dyn 223: 414-418, 2002.

6. Yang LV, Wan J, Ge Y, Fu Z, Kim SY, Fujiwara Y, Taub JW, Matherly LH, Eliason J and Li L: The GATA site-dependent hemogen promoter is transcriptionally regulated by GATA 1 in hematopoietic and leukemia cells. Leukemia 20: 417-425, 2006.

7. Li CY, Zhan YQ, Xu CW, Xu WX, Wang SY, Lv J, Zhou Y, Yue PB, Chen B and Yang XM: EDAG regulates the proliferation and differentiation of hematopoietic cells and resists cell apoptosis through the activation of nuclear factor-kappa B. Cell Death Differ 11: 1299-1308, 2004.

8. Tobler A, Moser B, Dewald B, Geiser T, Studer H, Baggiolini M and Fey MF: Constitutive expression of interleukin-8 and its receptor in human myeloid and lymphoid leukemia. Blood 82: 2517-2525, 1993.

9. Hedvat CV, Yao JJ, Sokolic RA and Nimer SD: Myeloid ELF1like factor is a potent activator of interleukin-8 expression in hematopoietic cells. J Bio Chem 279: 6395-6400, 2004.

10. Steube KG, Meyer C and Drexler HG: Multiple regulation of constitutive and induced interleukin 8 secretion in human myelomonocytic cell lines. Cytokine 12: 1236-1239, 2000. 
11. Mazur B, Mertas A, Sonta-Jakimczyk D, Szczepanski T and Janik-Moszant A: Concentration of IL-2, IL-6, IL-8, IL-10 and TNF-alpha in children with acute lymphoblastic leukemia after cessation of chemotherapy. Hematol Oncol 22: 27-34, 2004.

12. Faderl S, Do KA, Johnson MM, Keating M, O'Brien S, Jilani I, Ferrajoli A, Ravandi-Kashani F, Aguilar C, Dey A, Thomas DA, Giles FJ, Kantarjian HM and Albitar M: Angiogenic factors may have a different prognostic role in adult acute lymphoblastic leukemia. Blood 106: 4303-4307, 2005.

13. Hatfield KJ, Olsnes AM, Gjertsen BT and Bruserud O: Antiangiogenic therapy in acute myelogenous leukemia: targeting of vascularendothelial growth factor and interleukin 8 as possible antileukemic strategies. Curr Cancer Drug Targets 5: 229-248, 2005.

14. Galdiero M, Galdiero M, Finamore E, Rossano F, Gambuzza M, Catania MR, Teti G, Midiri A and Mancuso G: Interleukin-8 production by THP-1 cells stimulated by Salmonella enterica serovar Typhimurium porins is mediated by $\mathrm{AP}-1, \mathrm{NF}-\mathrm{\kappa B}$ and MAPK pathways. Cytokine 27: 15-24, 2004.

15. Kim MS, Lim WK, Park RK, Shin T, Yoo YH, Hong SH, An NH and Kim HM: Involvement of mitogen-activated protein kinase and NF- $\kappa \mathrm{B}$ activation in $\mathrm{Ca}^{2+}$-induced IL- 8 production in human mast cells. Cytokine 32: 226-233, 2005.

16. Secchiero P, Corallini F, Barbarotto E, Melloni E, di Iasio MG, Tiribelli $\mathrm{M}$ and Zauli G: Role of the RANKL/RANK system in the induction of interleukin-8 (IL-8) in B chronic lymphocytic leukemia (B-CLL) cells. J Cell Physiol 207: 158-164, 2006.

17. Kyriazis AP, Kyriazis AA, Scarpelli DG, Fogh J, Rao MS and Lepera R: Human pancreatic adenocarcinoma line Capan-1 in tissue culture and the nude mouse: morphologic, biologic, and biochemical characteristics. Am J Pathol 106: 250-260, 1982.

18. Lu J, Xu WX, Wang SY, Jiang Y, Li CY, Cai WM and Yang XM: Overexpression of EDAG-1 in NIH3T3 cells leads to malignant transformation. Sheng Wu Hua Xue Yu Sheng Wu Wu Li Xue Bao 34: 95-98, 2002.

19. Zhou SH, He DC, Zhang LJ and Chen LD: Prokaryotic expression of fusion protein TAT-EDAG and study on its transduction activity. Sheng Wu Gong Cheng Xue Bao 22: 598-603, 2006 (In Chinese).
20. An LL, Li G, Wu KF, Ma XT, Zheng GG, Qiu LG and Song YH: High expression of EDAG and its significance in AML. Leukemia 19: 1499-1502, 2005.

21. Emadi S, Clay D, Desterke C, Guerton B, Maquarre E, Charpentier A, Jasmin C and Le Bousse-Kerdiles MC: IL-8 and its CXCR1 and CXCR2 receptors participate in the control of megakaryocytic proliferation, differentiation, and ploidy in myeloid metaplasia with myelofibrosis. Blood 105: 464-473, 2005.

22. Li A, Dubey S, Varney ML, Dave BJ and Singh RK: IL-8 directly enhanced endothelial cell survival, proliferation, and matrix metalloproteinases production and regulated angiogenesis. J Immunol 170: 3369-3376, 2003.

23. Aguayo A, Giles F and Albitar M: Vascularity, angiogenesis and angiogenic factors in leukemias and myelodysplastic syndromes. Leuk Lymphoma 44: 213-222, 2003

24. Vinante F, Rigo A, Tecchio C, Patuzzo C, Ricetti MM, Morosato L, Cassatella MA, Chilosi M and Pizzolo G: Preferential release of high amounts of interleukin- 8 by myeloid blasts showing monocytic differentiation. Haematologica 81: 195-200, 1996.

25. Vinante F, Rigo A, Vincenzi C, Ricetti MM, Marrocchella R, Chilosi M, Cassatella MA, Bonazzi L and Pizzolo G: IL-8 mRNA expression and IL-8 production by acute myeloid leukemia cells. Leukemia 7: 1552-1556, 1993.

26. Vinante F, Rigo A, Cassatella MA and Pizzolo G: Interleukin-8 in acute myeloid leukemia. Blood 93: 1437, 1999.

27. Griffin JD: Leukemia stem cells and constitutive activation of NF-kappaB. Blood 98: 2291, 2001.

28. Horie R, Watanabe T and Umezawa K: Blocking NF-kappaB as a potential strategy to treat adult T-cell leukemia/lymphoma. Drug News Perspect 19: 201-209, 2006.

29. Pei XH, Nakanishi Y, Inoue H, Takayama K, Bai F, Inoue K, Harada $\mathrm{T}$ and Hara N: Polycyclic aromatic hydrocarbons induce IL-8 expression through nuclear factor B activation in A549 cell line. Cytokine 19: 236-241, 2002. 